Journal of Artificial Intelligence 5 (3): 99-112, 2012

ISSN 1994-5450 / DOI: 10.3923/jai.2012.99.112

(C) 2012 Asian Network for Scientific Information

\title{
A Genetic Algorithms-based Procedure for Automatic Tolerance Allocation Integrated in a Commercial Variation Analysis Software
}

\author{
L. Governi, R. Furferi and Y. Volpe \\ Dipartimento di Meccanica e Tecnologie Industriali, Universita degli Studi di Firenze, Via di Santa Marta, \\ 3, 50139 Firenze, Italy
}

Corresponding Author: L. Governi, Dipartimento di Meccanica e Tecnologie Industriali, Universita degli Studi di Firenze, Via di Santa Marta, 3, 50139 Firenze, Italy Tel: +390554796396

\begin{abstract}
In the functional design process of a mechanical component, the tolerance allocation stage is of primary importance to make the component itself responding to the functional requirements and to cost constraints. Present state-of-the-art approach to tolerance allocation is based on the use of Statistical Tolerance Analysis (STA) software packages which, by means of Monte Carlo simulation, allow forecasting the result of a set of user-selected geometrical and dimensional tolerances. In order to completely automate and optimize this process, this work presents a methodology to allow an automatic tolerance allocation, capable to minimize the manufacturing cost of a single part or assembly. The proposed approach is based on the Monte Carlo method to compute the statistical distribution of the critical to quality characteristics and uses an optimization technique based on Genetic Algorithms. The resulting procedure has been integrated in an off-the-shelf variation analysis software: eM-TolMate (by Siemens AG). Both the description of the optimization algorithm and some practical applications are presented in order to demonstrate the effectiveness of the proposed methodology.
\end{abstract}

Key words: Functional design, genetic algorithms, Monte Carlo method, tolerance allocation

\section{INTRODUCTION}

The design methods based on the concurrent engineering philosophy always demand for more powerful computer tools, capable of reducing both the cost and the development time of new products. In mechanical design, the tolerance allocation stage is one of the most demanding in terms of time. Such operation is normally carried out manually by an iterative process, based on subjective criterions and on the designer's skill (Ramani et al., 1998).

Several commercial software packages are available to help the designer in the evaluation of a given set of tolerance (or configuration) of a mechanical part or assembly. These software packages facilitate an accurate analysis of a user defined tolerance set in order to validate it on the basis of the functional requirements the part or the assembly must satisfy. However, there are only a few tools capable of performing an automatic tolerance choice, according to functional requirements and cost criteria. Furthermore, they work on simple problems involving only dimensional tolerances (Bjorke, 1978). In this work, a software tool will be presented which has been integrated in variation analysis software (eM-TolMate) and is capable to automatically perform an optimized tolerance allocation for any kind of mechanical assemblies. 
In commercial variation analysis software packages, the functional requirements a mechanical assembly has to fulfill are usually expressed by means of control measurements between different parts of the assembly. In order to choose the proper optimization technique, due to the unfeasibility of determining the mathematical relationship between the tolerances and the control measurements, a thorough investigation on the optimization methods has to be carried out (Chase et al., 1995).

Many optimization techniques have been applied to the automatic tolerance allocation problem; the Lagrange Multiplier method (Rajasekera and Fang, 1995), the artificial neural networks (Kopardekar and Anand, 1995; Al-Mohammed et al., 2001), the fuzzy logic (Kopardekar and Anand, 1995; Mao et al., 2009) and the genetic algorithms (Kanai et al., 1995; Singh et al., 2004) are a few examples of such techniques.

On the basis of both our previous experiences and the characteristics of the problem, we chose the Genetic Algorithms (GA) heuristic technique which is described by Al-Bastaki and Awad (2010).

The idea of combining the GA with Monte Carlo analysis has been used by Lee (1993) and by Iannuzzi and Sandgren (1994). In this study, several modifications to Iannuzzi's approach are proposed and evaluated.

Moving from these considerations, the main aim of this study is to describe a new methodology to provide an automatic tolerance allocation, capable of minimizing the manufacturing cost of a single part or assembly. The newly devised procedure allows setting the variability range for each tolerance, the cost model chosen for the corresponding manufacturing process and an objective maximum variability range for a set of user selected Control Measurements (CMs). In particular, several tolerance configurations are analyzed by means of the Monte Carlo method (integrated in eM-TolMate) coupled with the GA-based procedure; on the basis of the obtained results, the configuration satisfying the functional requirements and minimizing the manufacturing cost is attained.

\section{MATERIALS AND METHODS}

The proposed approach for automatic tolerance allocation is based on a GA oriented procedure, implemented and integrated in the commercial software package eM-TolMate. As widely known (Goldberg, 1989), GA is an optimization technique based on the biological genetic processes which simulates the natural evolution: the individuals which are more suitable to the environment (fit individuals) have more opportunities to survive and reproduce themselves; the others will die and will probably have no children.

In this study, each GA population is formed by a number of tolerance configurations describing the problem to be analyzed. The GA procedure starts working from an initial random population and derives the following ones by means of the classical GA operators, working according to a standard five-step procedure:

Step 1: Encoding of the design variables. In this step, the values of the design variables representing the individual are encoded into a binary string; the combinations of all the possible design variables make up the algorithm's search space (Mukhopadhyay et al., 2009)

Step 2: Computation of the Objective Function (OF). In this step, each individual's performance is calculated; the performance is expressed by OF value fitness score

Step 3: Selection of the best-performing individuals. In this step, the best performing individuals are preserved and the worst performing ones are discarded according to a statistical criterion 


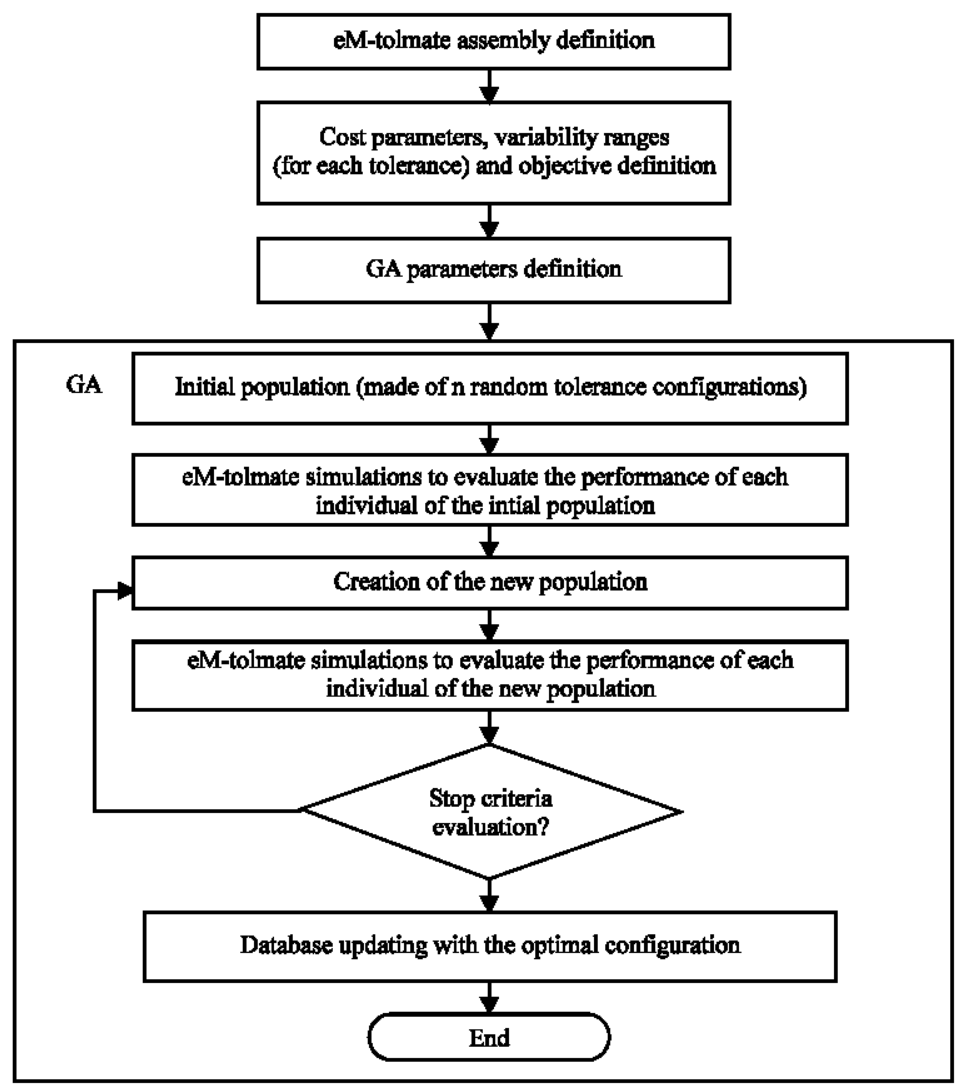

Fig. 1: GA flow chart

Step 4: Crossover. In this step, the individual's subparts are mixed so that new individuals are born in order to improve the whole population

Step 5: Mutation. In this step, variability is introduced to prevent early algorithm convergence and to guarantee a sufficient exploration of the design space

The tolerance allocation procedure works according to the block diagram shown in Fig. 1. The GA are a very robust technique and do not necessitate the formal knowledge of the function to be maximized but only a way of computing its value for a given set of variables values (Alma and Bulut, 2012).

eM-TolMate: The variation analysis software eM-TolMate, which is described and reviewed in Chiesi and Governi (2003), simulates the assembly procedures of mechanical parts and enables the user to define a complete tolerance configuration both using ASME and ISO standards (statistical tolerancing can be used). The tolerances are defined by means of eM-TolMate features (planes, holes, pins, etc.) which rely, in turn, on the CAD model of the mechanical part. The definition of the CMs is performed on the basis of the previously defined features; the requirements on the CMs are expressed by means of the well-known statistical coefficients $\mathrm{C}_{\mathrm{p}}$ and $\mathrm{C}_{\mathrm{pk}}$ which are related to process capability.

eM-TolMate computes the statistical distributions of the CMs and provides, if required, a contribution analysis indicating the tolerances more remarkably affecting them. eM-TolMate 
performs the tolerance analysis by means of the Monte Carlo method and can carry out the simulation even if an analytical relationship between the input variables and the output one is not available. In this study, eM-TolMate is the tool which serves to compute the OF value (i.e., the performance) of each single tolerance configuration generated by the GA.

For a generic tolerance configuration, each CM is estimated by means of its mean value, standard deviation of its statistical distribution and indexes $\mathrm{C}_{\mathrm{p}}$ and $\mathrm{C}_{\mathrm{pk}}$; on the basis of the computed values, the corresponding OF value is calculated according to the formulations described below.

The problem definition and the tolerances data: The first step of the procedure consists of the usual definition of the CAD model and of the assembly tree; secondarily, it is required to state the upper and lower limits for each tolerance. Such limits represent the research space of the optimization procedure and depend on the different manufacturing process involved in the achievement of each tolerance.

After that, it is necessary to define all the parameters related to the computation of the cost associated to each single tolerance. Several cost models can be found in the scientific literature; all of them estimate the cost associated to a certain tolerance by a function which can be more or less complex (Wu et al., 1998), decreasing while the tolerance value increases. As far as a comprehensive investigation on the cost models is not the primary objective of this work, in order to validate the proposed routines we used Hillier's cost model which evaluates the cost associated to a tolerance simply assuming that it is inversely proportional to the second power of its amplitude. Consequently the overall cost of a set of tolerances with amplitudes $t_{i}$ is provided by the following equation:

$$
\mathrm{C}_{\mathrm{tot}}=\sum \frac{\mathrm{a}_{\mathrm{i}}}{\mathrm{t}_{\mathrm{i}}^{2}}
$$

where, the coefficients $a_{i}$ are user defined coefficients which are related to the manufacturing process.

The automatic allocation procedure allows the user to define the cost models which more closely fit the company data and to choose different kinds of tolerances:

- Tolerances continuously varying inside a user defined range

- Tolerances which may assume only discrete values defined by the user

Together with the tolerances, also the CMs data must be defined; for each of them it is requested to choose:

- The allowed upper limit value

- The allowed lower limit value

- The objective $\mathrm{C}_{\mathrm{pk}}$ value which, at least, must be achieved for the given CM

The objective function definition: The functional requirements in a mechanical component or assembly design may be represented by a number of control measurements whose values must lay into a specified range. Once such measurements are defined, the user is asked to set, for each of 
them, the acceptable variability range. Each range corresponds to the objective tolerance on the correspondent CM $\left(\mathrm{Obj}_{\mathrm{to}}\right)$. On this basis, by means of a Monte Carlo simulation (Hahn and Shapiro, 1994; Hossin et al., 2011), the capability of a given tolerance configuration to suit the target functional requirements can be expressed by the statistical coefficients $\mathrm{C}_{\mathrm{p}}$ and $\mathrm{C}_{\mathrm{pk}}$, computed with respect to the required $\mathrm{Obj}_{\mathrm{tol}}$ values. The $\mathrm{C}_{\mathrm{pk}}$ index, specifically, can provide a useful estimate even if the process statistical distribution is not centered.

The cost corresponding to each tolerance configuration is evaluated by means of the cost models selected for each tolerance. Throughout this work, several OF formulations have been devised and investigated.

The first one was based on the formulation proposed by Iannuzzi and Sandgren (1994):

$$
\text { fitness }=-\sum_{\mathrm{i}=1}^{N_{\mathrm{t}}} \frac{\mathrm{a}_{\mathrm{i}}}{\left(\mathrm{t}_{\mathrm{i}}\right)^{\mathrm{b}_{\mathrm{i}}}}+\mathrm{R} \sum_{\mathrm{j}=1}^{\mathrm{N}_{\mathrm{c}}}\left[\mathrm{g}_{\mathrm{j}}(\mathrm{t})\right]
$$

where, $a_{i}$ and $b_{i}$ are the cost coefficients chosen for the ith tolerance, $R$ is a penalty factor, $g_{j}$ is the function expressing the jth functional requirement. Such a relationship was found to be of little use, due to the lack of repeatability of the obtainable results: in some cases the algorithm reached excessively high quality configurations with a very high associated costs, in other cases, on the contrary, the configurations were characterized by a very low cost but exhibited a totally insufficient fulfillment of the functional requirements. This behavior can be explained by the strong dependency of the OF trend on the parameter R in Eq. 2. In order to obtain satisfactory results, both minimizing the total cost and fulfilling the functional requirements, it is necessary to recalibrate the parameter $R$ whenever a new mechanical assembly is analyzed, since in all our experiments no general value for parameter $\mathrm{R}$ could be determined. The calibration requires several runs of the allocation procedure on each new assembly, resulting in a totally unacceptable computation time.

The drawback of a repeated calibration of parameter $\mathrm{R}$ was overtaken by devising a new $\mathrm{OF}$ based on a hierarchical approach. Such an approach consists of computing the OF value by evaluating the $\mathrm{N}_{\mathrm{o}}$ terms in Eq. 2, sequentially: once a tolerance configuration satisfying the jth functional requirement is found, the optimization algorithm seeks a new configuration satisfying both the $1 \mathrm{st}$, $2 \mathrm{nd}, \ldots$, jth requirement and the $(j+1)$ th. Only after all the terms in the second summation of Eq. 1 are computed, the term depending on the cost is added (first summation in Eq. 2).

The allocation procedure using the new OF proved to be capable of providing completely satisfactory tolerance configuration (satisfaction of all the functional requirements and minimum associated cost); nevertheless this behavior was maintained only for simple case studies (20 tolerances, $5 \div 6 \mathrm{CMs}$ ). In the analysis of more complex cases (100 tolerances, $10 \div 15 \mathrm{CMs}$ ), the OF does not guarantee the convergence of the allocation procedure to a suitable solution; in the case a satisfactory tolerance configuration is found, the repetition of the allocation procedure often leads to considerably different results, usually satisfying the functional requirements but with a very high associated cost. The main cause for such a behavior is the increasing number of variables which the GA has to take into account and which prevents a comprehensive exploration of the search space. According to the described results, a further modification in the OF definition was introduced. In the new OF, the evaluation of the functional requirements takes place for all the CMs at the same time, while the cost factor is added only if all the requirements are satisfied, as shown in the block diagram of Fig. 2. 


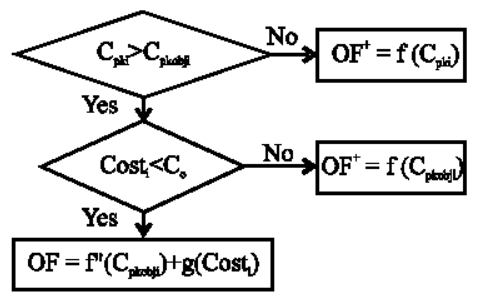

Fig. 2: Final objective function block diagram

Introduction of advanced operators in the GA: A further improvement of the performance of the automatic tolerance allocation procedure was achieved by the introduction of several advanced operators in the main structure of the GA.

Firstly, an auxiliary mutation operator was devised, working together with the classical GA mutation. Such an operator is meant to introduce the necess ary variability in the set of tolerance configurations but, unlike the classical operator, may vary a given tolerance configuration only changing it into a new one characterized by wider tolerance values and consequently by a lower overall cost. This effect is obtained simply allowing the mutation of zeros into ones in the binary string representing a tolerance configuration but not vice versa. The new operator enables the GA to explore more completely the search space, with particular reference to the individuals characterized by a lower cost, thereby improving the overall performance of the algorithm.

Secondarily, a variable mutation probability was introduced for the classical mutation operator. The variable mutation probability, according to the model proposed by Zimmermann et al. (1999), depends on:

- The user selected lower and upper limits for the mutation probability $\left(\mathrm{pm}_{1}\right.$ and $\mathrm{pm}_{2}$, respectively)

- The average diversity coefficient (rate) D among the individuals of a population

According to Zimmermann's definition, if the individuals belonging to the same population are very similar to the others, the value of the coefficient $D$ is close to 1 , otherwise tends to 0 .

The opportunity of linking the mutation probability value to the average diversity $\mathrm{D}$ proved to be very useful; in fact, in such a way, the mutation operator is used as much as the GA demands for it. In the initial phase, when the GA is mainly exploring the search space, the mutation probability value should be low because the characteristics of the best performing individuals have not spread through the population yet; on the other side, as the number of generations analyzed by the GA grows, the mutation probability value must increase, since the variety of the "genetic patrimony" is low.

The relationship for the computation of the mutation probability permutation is provided in Eq. 3:

$$
\mathrm{p}_{\text {mutation }}=\mathrm{pm}_{1} \cdot \exp \left(\mathrm{D} \cdot \ln \frac{\mathrm{pm}_{2}}{\mathrm{pm}_{1}}\right)
$$

It is evident that, in the case $\mathrm{D}=0$, then $\mathrm{p}_{\text {mutation }}=\mathrm{pm}_{1}$; otherwise, if $\mathrm{D}=1$, then $\mathrm{p}_{\text {mutation }}=\mathrm{pm}_{2}$. 


\section{RESULTS AND DISCUSSION}

The results obtained by several test runs on mechanical assemblies characterized by a different complexity degree, show that the combination of the OF, described in Fig. 2, with the additional mutation operator and the variable mutation probability, guarantees the achievement of satisfactory results (satisfaction of the functional requirements and minimum cost) for any mechanical assembly of practical interest. To prove this claim, four examples of different mechanical assemblies have been analyzed with the aim of pointing out the behavior of the allocation procedure. For each example, the reference tolerance configuration which is the one to be compared with the optimized tolerance set achieved by the GA based procedure, is obtained by means of the well-known manual optimization process consisting in the contribution analysis and subsequent modification of the tolerance values until an optimum compromise between cost and functional requirements is reached.

In order to assess the performance of the devised procedure, a parameter, called "process capability difference" $\Delta \mathrm{C}_{\mathrm{pki}}^{\prime}$, is defined for each control measurement point.

In particular $\Delta \mathrm{C}_{\mathrm{pki}}^{\prime}$ (where $\mathrm{i}=1, \ldots \mathrm{n}$ is the number of control measurement points) is defined as the difference between the objective values (for each CM) and the optimized ones for any $\mathrm{C}_{\mathrm{pki}}$. By definition of $\mathrm{C}_{\mathrm{pk}}$, when $\Delta \mathrm{C}_{\mathrm{pki}}>0$ a better tolerance allocation for the ith $\mathrm{CM}$ is achieved. On the other hand, when the tolerances fall outside of the specification limits, $\Delta \mathrm{C}_{\mathrm{pki}}^{\prime}>0$. Finally, $\Delta \mathrm{C}_{\mathrm{pki}}>0$ when the obtained configuration (optimized) provides the same process capability of the objective tolerance allocation.

Application to a schematic crank motor and its support parts: The first devised example, a schematic assembly of a crank motor and its support parts, is shown in Fig. 3. The complete eM-TolMate model consists of 5 elements and 9 tolerances. Though, it is not a real industrial case study, this simple example can be used to emphasize some peculiarities of the GA-based procedure developed in this study.

The only functional requirement is represented by the CM between the crank and the plane on which the motor support lays (i.e., a minimum clearance is requested); the functional relationship between the tolerances and the CM is unknown.

The upper and lower limits for all the tolerance variability range (Zhang and Huq, 1992) are assumed to be respectively 0.01 and $1.00 \mathrm{~mm}$.

The objective $\mathrm{C}_{\mathrm{pk}}$ value is assumed to be 1.5. The initial tolerance configuration (see first column of Table 1) is obtained, using eM-TolMate, by a classical allocation procedure consisting of a set of user selected tolerance values and a subsequent statistical verification of the achieved performance

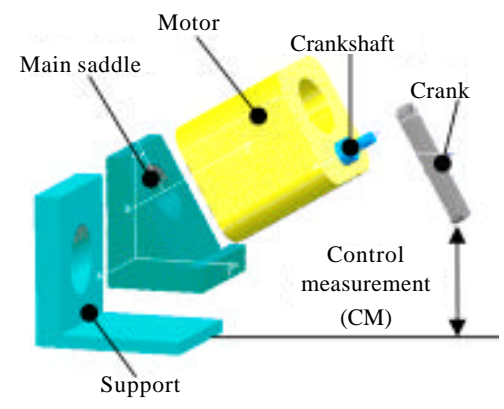

Fig. 3: Schematic assembly of a crank motor 
Table 1: Initial and optimized values for the crank motor assembly

\begin{tabular}{lllll}
\hline Objective values & Initial values & Optimized values & $\Delta \mathrm{C}_{\mathrm{pki}}^{\prime}$ & $\Delta \mathrm{C}_{\mathrm{pk}}^{\prime}>0$ \\
\hline $\mathrm{C}_{\mathrm{pk} 1}=1.50$ & $\mathrm{C}_{\mathrm{pk} 1}=1.10$ & $\mathrm{C}_{\mathrm{pk} 1}=1.51$ & 0.01 & Yes \\
Approximately equal to the initial cost & $\mathrm{Cost}=700.5$ & $\mathrm{Cost}=950.6$ & & \\
Cost reduction (\%) & -35.7 & & & \\
\hline
\end{tabular}

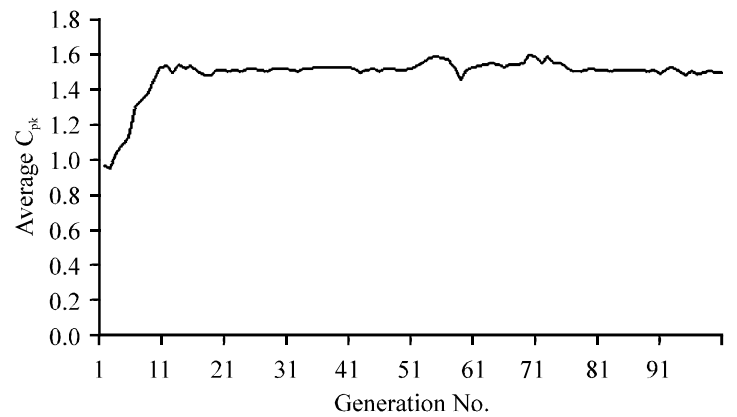

Fig. 4: Average trend vs. generation number

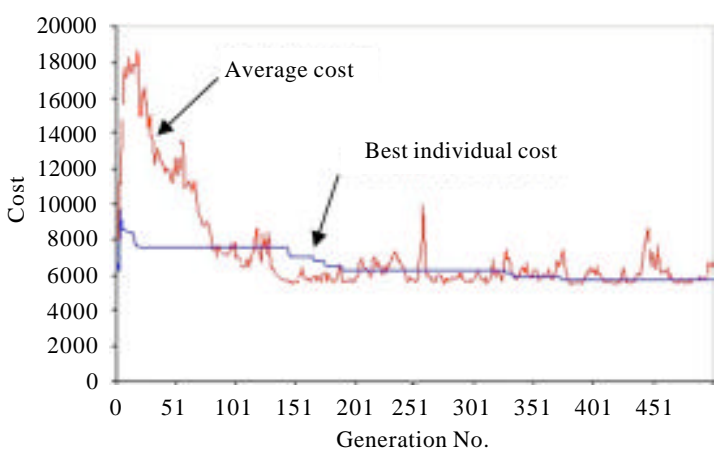

Fig. 5: $\mathrm{C}_{\mathrm{pk}}$ and cost trend vs. generation number

(i.e., manual check of the $\mathrm{CM}$ value). The $\mathrm{C}_{\mathrm{pk}}$ value for the initial tolerance configuration results equal to 1.1; the associated cost corresponding to each tolerance $\mathrm{t}_{\mathrm{i}}$ (evaluated according to the Eq. 1 where all the $a_{i}$ coefficients are assumed to be equal to 1), results equal to 700.5 .

In Fig. 4, the trend of the average $\mathrm{C}_{\mathrm{pki}}$ as the generation number of the GA increases is shown. For each generation, the average cost and the cost associated to the best individual are respectively depicted in Fig. 5. In the first generations the average $\mathrm{C}_{\mathrm{pki}}$ is quite low, meaning that the functional requirement has not been reached; as the allocation procedure goes on, after the 10th generation, the $\mathrm{C}_{\mathrm{pk} 1}$ value is approximately equal to the target one but never gets too high because of the cost term which tends to make the tolerance values low.

On the other side, in the first generations, the cost is very high because a configuration satisfying the functional requirements is yet to be found. As soon as this occurs, the cost, as expected (Rho and March, 1997), tends to decrease, approaching its final minimum value, corresponding to $\mathrm{C}_{\mathrm{pk} 1}=1.51$.

As depicted in Table 1, the automatic tolerance allocation procedure allows to obtain a new configuration characterized by $\Delta \mathrm{C}_{\mathrm{pk} 1}=0.01$. This means that the functional requirements are fulfilled even slightly better than desired. The difference between the objective values and the 

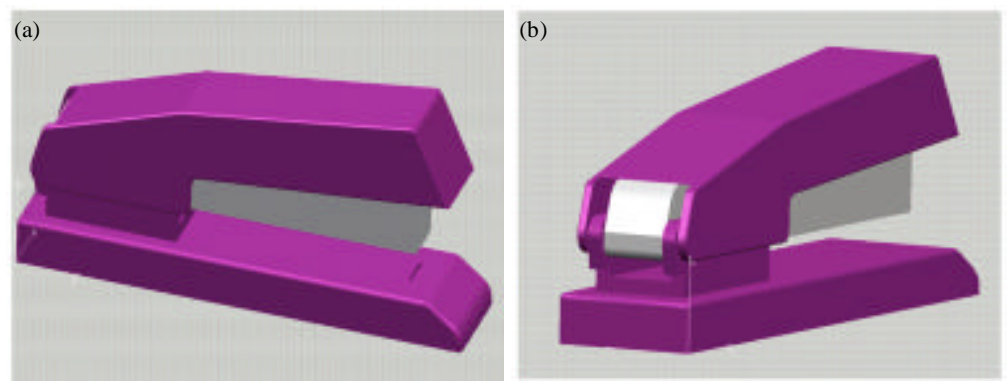

Fig. 6(a-b): Assembly of a stapler, (a) Upper side view and (b) Rear right view

Table 2: Initial and optimized values for the stapler assembly

\begin{tabular}{lllll}
\hline Objective values & Initial values & Optimized values & $\Delta \mathrm{C}_{\mathrm{pk}}^{\prime}$ & $\Delta \mathrm{C}_{\mathrm{pk}}^{\prime}>0$ \\
\hline $\mathrm{C}_{\mathrm{pk} 1}=0.50$ & $\mathrm{C}_{\mathrm{pk} 1}=0.51$ & $\mathrm{C}_{\mathrm{pk} 1}=0.54$ & 0.04 & Yes \\
$\mathrm{C}_{\mathrm{pk} 2}=1.00$ & $\mathrm{C}_{\mathrm{pk} 2}=1.10$ & $\mathrm{C}_{\mathrm{pk} 2}=1.01$ & 0.01 & $\mathrm{Yes}$ \\
<than initial cost & $\mathrm{Cost}=8037850$ & $\mathrm{Cost}=52859$ & & \\
Cost reduction (\%) & 99.3 & & & \\
\hline
\end{tabular}

initial values obtained using eM-TolMate results equal to -0.4, i.e., the initial value is out of functional specifications. On the other hand, in order to fulfil the user requirements, a $36 \%$ higher cost, equal to 950.6, proves to be necessary.

Application to the assembly of a stapler: The second case study is the assembly of a stapler, made of 3 components (Fig. 6). In this example there are $2 \mathrm{CMs}$ and 13 tolerances; unlike the previous assembly, the limits and the cost coefficients for each tolerance are assumed to be different.

The objective values for the two $\mathrm{C}_{\mathrm{pki}}$, shown in Table 2 , are assumed to be equal to 0.5 and 1.0 , respectively. The classical manual procedure, carried out using eM-TolMate, provides $\mathrm{C}_{\mathrm{pki}}$ values equal to 0.51 and 1.1 , respectively.

Using the proposed procedure, the new, optimized values for the two CMs result to be equal to 0.54 and 1.01 , respectively. This demonstrates that the automatic tolerance procedure allows on one side a considerable cost reduction (equal to 99.3\%) and, on the other hand, the tolerance configuration is acceptable since $\Delta \mathrm{C}_{\mathrm{pk} 1}^{\prime}$ and $\Delta \mathrm{C}_{\mathrm{pk} 2}^{\prime}$ are both greater than 0 .

The obtained results are in line with the ones coming from other studies using GA in mechanical (Singh et al., 2005) and in software development fields (Ghiduk and Girgis, 2010) where a cost reduction of more than $75 \%$ was reached by using approaches based on GA.

Application to the assembly of a PC mouse: The third, more complex, assembly consists of a $\mathrm{PC}$ mouse and is made of 6 different parts (Fig. 7a, b). Figure 7c shows the eM-TolMate assembly tree for the model.

The tolerance set consists of 34 different tolerances: 6 dimensional tolerances (linear dimensions and diameters), 7 flatness tolerances, 1 parallelism tolerance, 2 concentricity tolerances, 15 position tolerances and 3 profile tolerances. The optimization objective takes into account 6 different control measurements simultaneously (Fig. 7a, b):

CM 1: Board to base gap, it is necessary in order to correctly assemble the board inside the base 
(a)

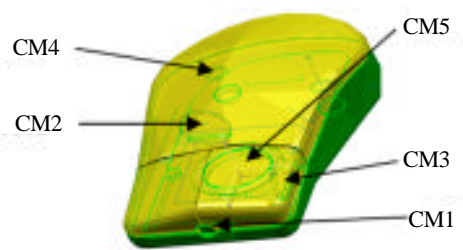

(c)

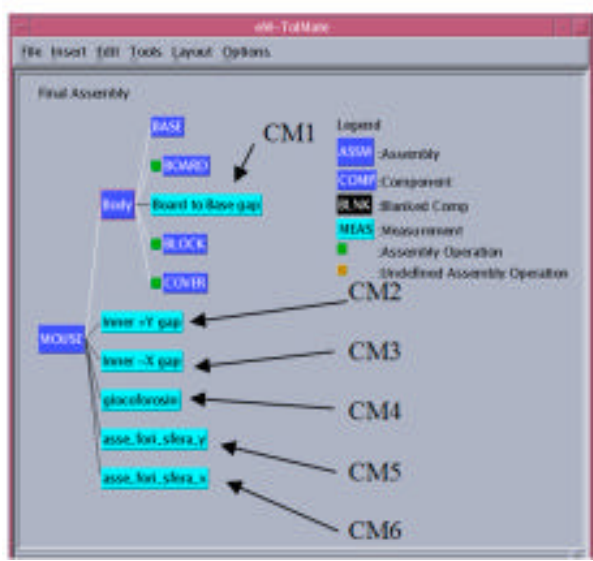

(b)

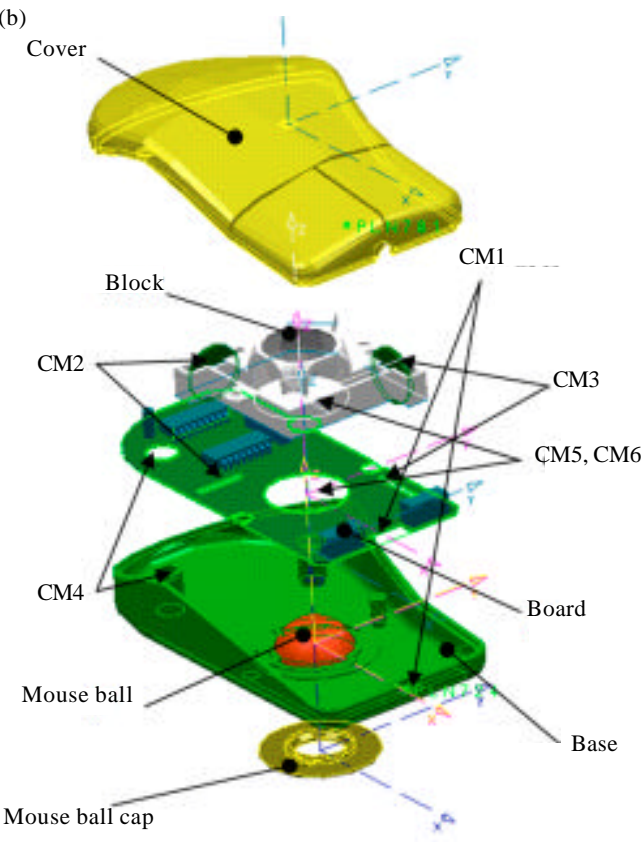

Fig. 7(a-b): (a) Mouse model, (b) Assembly parts and (c) Assembly tree, CM: Control measurement point

Table 3: Initial and optimized values for the mouse assembly

\begin{tabular}{lllll}
\hline Objective values & Initial values & Optimized values & $\Delta \mathrm{C}_{\mathrm{pk}}^{\prime}$ & $\Delta \mathrm{C}_{\mathrm{pk}}^{\prime}>0$ \\
\hline $\mathrm{C}_{\mathrm{pk} 1}=1.00$ & $\mathrm{C}_{\mathrm{pk} 1}=1.00$ & $\mathrm{C}_{\mathrm{pk} 1}=1.03$ & 0.02 & Yes \\
$\mathrm{C}_{\mathrm{pk} 2}=1.20$ & $\mathrm{C}_{\mathrm{pk} 2}=1.20$ & $\mathrm{C}_{\mathrm{pk} 2}=1.20$ & 0.00 & No \\
$\mathrm{C}_{\mathrm{pk} 3}=1.20$ & $\mathrm{C}_{\mathrm{pk} 3}=1.20$ & $\mathrm{C}_{\mathrm{pk} 3}=1.23$ & 0.02 & $\mathrm{Y}$ \\
$\mathrm{C}_{\mathrm{pk} 4}=1.50$ & $\mathrm{C}_{\mathrm{pk} 4}=1.50$ & $\mathrm{C}_{\mathrm{pk} 4}=1.50$ & 0.02 & $\mathrm{Yes}$ \\
$\mathrm{C}_{\mathrm{pk} 5}=1.50$ & $\mathrm{C}_{\mathrm{pk} 5}=1.50$ & $\mathrm{C}_{\mathrm{pk} 5}=1.51$ & 0.02 & $\mathrm{Yes}$ \\
$\mathrm{C}_{\mathrm{pk} 6}=1.50$ & $\mathrm{C}_{\mathrm{pk5} 5}=1.50$ & $\mathrm{C}_{\mathrm{pk5}}=1.54$ & 0.02 & $\mathrm{Yes}$ \\
$<$ than initial cost & $\mathrm{Cost}=1747.9$ & $\mathrm{Cost}=753.0$ & & \\
Cost reduction (\%) & 56.9 & & & \\
\hline
\end{tabular}

CM 2 and CM 3: Guiding wheels clearances, they provide the mouse pointing functionality CM 4:

Base pin-board hole clearance, necessary in order to correctly assemble the board inside the base

CM 5 and CM 6: Board and block holes, they account for the $\Delta \mathrm{x}$ and $\Delta \mathrm{y}$ position between the two holes centers

Like in the previous example, the upper and lower limits vary according to the tolerance type. In this example the objective values are set equal to the ones obtained by using the manual procedure. Table 3 shows that for all the CMs (except for CM 2 whose $\mathrm{C}_{\mathrm{pk}}$ remains unchanged and equal to the optimization target) $\Delta \mathrm{C}_{\mathrm{pki}}^{\prime}>0$. As a consequence, once more, almost all the objective $\mathrm{C}_{\mathrm{pk}}$ values have exceeded the optimization objectives, with a much lower manufacturing cost (59.6\% of the initial one). 

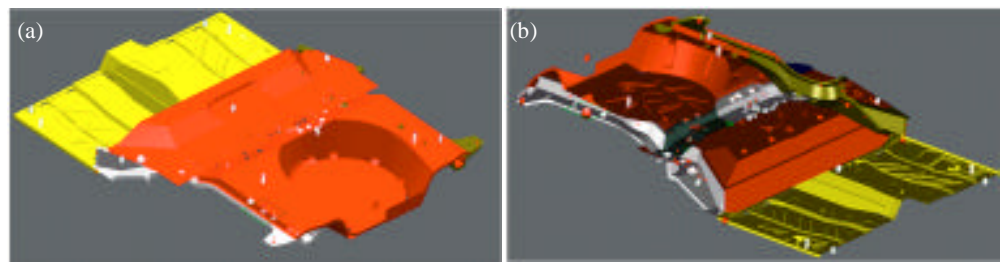

Fig. 8(a-b): Car floor model, (a) Upper left view and (b) Lower right view

Table 4: Initial and optimized values for the car floor

\begin{tabular}{|c|c|c|c|c|}
\hline Objective values & Initial values & Optimized values & $\Delta \mathrm{C}_{\mathrm{pk}}^{\prime}$ & $\Delta \mathrm{C}_{\mathrm{pk}}^{\prime}>0$ \\
\hline $\mathrm{C}_{\mathrm{pk} 1}=1.00$ & $\mathrm{C}_{\mathrm{pk} 1}=1.00$ & $\mathrm{C}_{\mathrm{pk} 1}=1.10$ & 0.00 & No \\
\hline $\mathrm{C}_{\mathrm{pk} 2}=1.00$ & $\mathrm{C}_{\mathrm{pk} 2}=1.00$ & $\mathrm{C}_{\mathrm{pk} 2}=1.01$ & 0.04 & Yes \\
\hline $\mathrm{C}_{\mathrm{pk} 3}=1.30$ & $\mathrm{C}_{\mathrm{pk} 3}=1.30$ & $\mathrm{C}_{\mathrm{pk} 3}=1.32$ & 0.02 & Yes \\
\hline $\mathrm{C}_{\mathrm{pk} 4}=0.65$ & $\mathrm{C}_{\mathrm{pk} 4}=0.65$ & $\mathrm{C}_{\mathrm{pk} 4}=0.66$ & 0.04 & Yes \\
\hline $\mathrm{C}_{\mathrm{pk} 5}=1.00$ & $\mathrm{C}_{\mathrm{pk} 5}=1.00$ & $\mathrm{C}_{\mathrm{pk} 5}=1.10$ & 0.00 & Yes \\
\hline $\mathrm{C}_{\mathrm{pk} 5}=0.90$ & $\mathrm{C}_{\mathrm{pk} 5}=0.90$ & $\mathrm{C}_{\mathrm{pk5} 5}=0.93$ & 0.02 & Yes \\
\hline $\mathrm{C}_{\mathrm{pk} 7}=0.95$ & $\mathrm{C}_{\mathrm{pk} 7}=0.95$ & $\mathrm{C}_{\mathrm{pk} 7}=0.95$ & 0.00 & No \\
\hline $\mathrm{C}_{\mathrm{pk} 8}=1.00$ & $\mathrm{C}_{\mathrm{pk} 8}=1.00$ & $\mathrm{C}_{\mathrm{pk} 8}=1.00$ & 0.00 & No \\
\hline $\mathrm{C}_{\mathrm{pk} 9}=1.00$ & $\mathrm{C}_{\mathrm{pk} 9}=1.00$ & $\mathrm{C}_{\mathrm{pk} 9}=1.00$ & 0.00 & No \\
\hline $\mathrm{C}_{\mathrm{pk} 10}=1.00$ & $\mathrm{C}_{\mathrm{pk} 10}=1.00$ & $\mathrm{C}_{\mathrm{pk} 10}=1.10$ & 0.00 & No \\
\hline$<$ than initial cost & Cost $=2536$ & Cost $=1751$ & & \\
\hline Cost reduction (\%) & 30.9 & & & \\
\hline
\end{tabular}

Application to the assembly of a car floor: The last example consists of a car floor (Fig. 8). The eM-TolMate model is made of 69 tolerances and $10 \mathrm{CMs}$. The limits for the tolerances values (gaps between sheet metal parts) have been selected according to the experience of a team of skilled operators working for a well-known car manufacturing company and, thereby, result to be realistic. The objective values for the functional requirements $\left(\mathrm{C}_{\mathrm{pki}}\right)$, shown in Table 4 , are set equal to the ones obtained by using the manual allocation procedure which provided the starting configuration.

By means of the automatic tolerance allocation procedure, better values have been reached for 5 out of $10 \mathrm{CMs}$ and the associated cost is considerably lower (30.9\%) as depicted in Table 4. The $\Delta \mathrm{C}_{\mathrm{pki}}$ for the remaining $5 \mathrm{CM}$ result equal to 0 (i.e., exactly equal to the optimization objective) thus proving that the optimized configuration is characterized, globally, by a better process capability with a lower associated cost.

Performance comparison with other methods: A comparison between the overall results of the proposed method and the ones obtainable by different ones described in the scientific literature is not straightforward (Chiong and Beng, 2007). This is due to the fact that, as anticipated above, almost all the authors present their results applied to different case studies, while a reliable comparison should be conducted on the same set of case studies. However, a qualitative comparison can be carried out considering that in all the works describing GA-based methods for tolerance allocation, a cost associated to the analyzed tolerance configuration is presented. From this point of view, the method described in this paper can be compared against the results obtained in such studies.

By way of example, to evaluate the performance of their proposed GA-based approach, Chen and Fischer (2000) compared 12 different cases solved by means of, respectively, GA and gradient 
based methods. The average absolute difference (in \%) between the cost function obtained using the two different methods resulted to be equal to about $2 \%$.

Kumar et al. (2009) compared the performance of their GA-based method with classical GA, Simulated Annealing and Complex research methods (Choi et al., 2000) demonstrating a reduction of the cost function in the range $20-27 \%$. Such a result is comparable with the one provided by Muthu et al. (2009) that demonstrate an average cost reduction of about $21 \%$ using their GA-based method tested against conventional optimization methods.

As depicted in the four examples discussed above, in the present work the average cost reduction between the new GA based method and the one obtained by using a classical tolerance allocation procedure resulted to be about $34.7 \%$. Usually, gradient based optimization techniques, like the ones used as a term of comparison in the works mentioned above, lead to results similar to those which can be obtained with manual optimization based on contribution analysis. As a consequence, the average cost reduction obtained by the method described in this work appears to be in line with the results described by other authors, thus proving that the proposed integrated tool is effective for automatically optimizing a tolerance set on a mechanical part or assembly.

\section{CONCLUSIONS}

In this study a novel approach, based on the Monte Carlo method coupled with a modified GA procedure for automatic tolerance allocation, has been described. Different formulations of the OF have been investigated and a number of advanced operators have been introduced in order to provide the necessary repeatability and accuracy. The approach proves to be capable of minimizing the manufacturing cost of a single part or assembly, as demonstrated by the analysis of four exemplificative case studies. The procedure allows a global approach to the tolerance allocation problem, so that all the tolerances can be effectively varied thereby reaching a configuration characterized by the fulfillment of all the user-defined functional requirements associated to an extremely low cost if compared to the well-known manual tolerance optimization process based on contribution analysis. The final result of this work is a software tool which is completely integrated in eM-TolMate (a commercial variation analysis software by Siemens AG) which can be of valuable help to the designer involved in the tolerance allocation process of mechanical assemblies. The presented software tool can be useful both in the initial phase of the work (i.e., when only the functional requirements have been stated) and in the final optimization stage, when a manually obtained tolerance configuration is already available.

\section{ACKNOWLEDGMENTS}

The authors are grateful to Tecnomatix Technologies (now Siemens AG) whose precious support allowed the implementation and the integration in eM-TolMate of essential parts of the automatic tolerance allocation procedure presented in this work. The authors would also like to acknowledge the support provided by FIAT Auto by the collaboration of production engineers involved in the practical process of tolerance allocation.

\section{NOMENCLATURE}

Cost $_{i}=$ Tolerance configuration cost

$\mathrm{C}_{\mathrm{pki}}, \mathrm{C}_{\mathrm{pi}}=$ Capability indexes

$\mathrm{C}_{\mathrm{pkobj}}=$ Objective $\mathrm{C}_{\mathrm{pk}}$ value for a control measurement

$\mathrm{C}_{\text {tot }}=$ Overall cost for the analysed mechanical assembly or part 


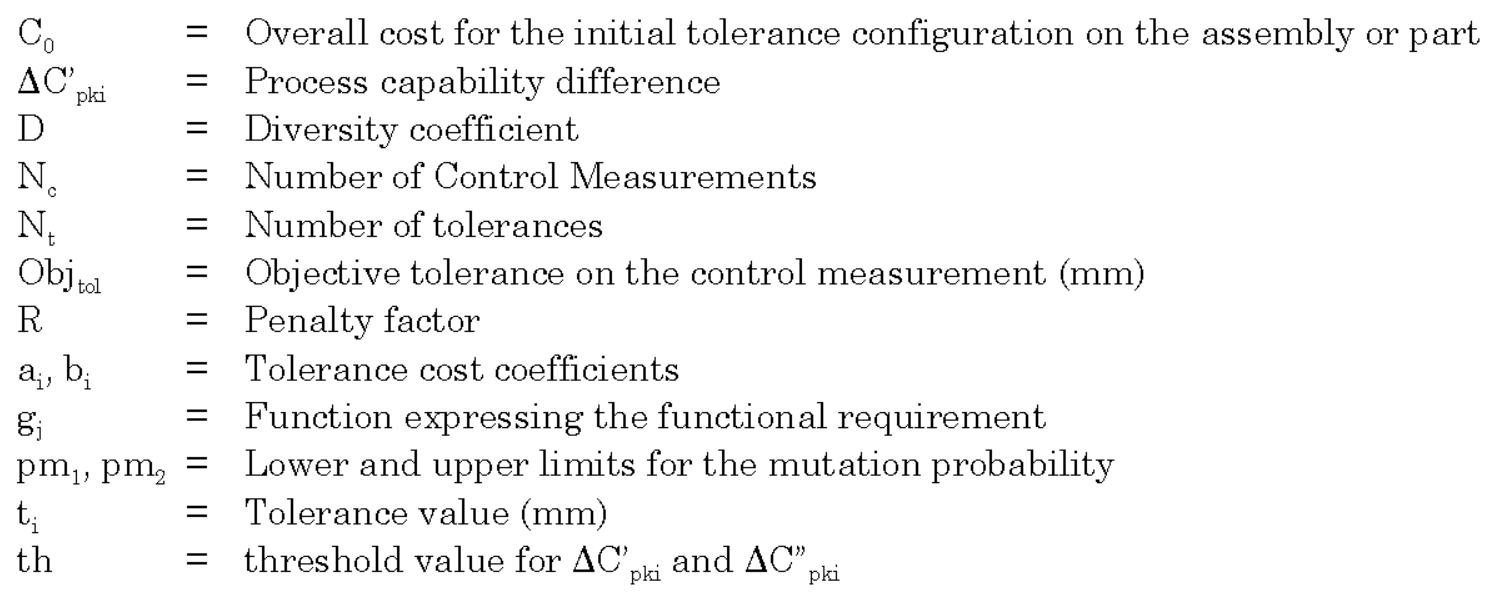

\section{REFERENCES}

Al-Bastaki, Y. and W. Awad, 2010. GADS and reusability. J. Artif. Intell., 3: 67-72.

Al-Mohammed, M., D. Esteve and J. Boucher, 2001. Tolerance allocation for an electronic system using neural network/Monte-Carlo approach. Proc. Inter. Soc. Opt. Eng., 4540: 446-457.

Alma O.G. and E. Bulut, 2012. Genetic algorithm based variable selection for partial least squares regression using ICOMP criterion. Asian J. Math. Stat., 5: 82-92.

Bjorke, O., 1978. Computer Aided Tolerancing. Tapir Publisher, Trondheim, Norway, ISBN: 9788251902526, Pages: 153.

Chase, K.W., J. Gao and S.P. Magleby, 1995. General 2-D tolerance analysis of mechanical assemblies with small kinematic adjustments. J. Design Manufact., 5: 263-274.

Chen, T.C. and G.W. Fischer, 2000. A GA-based search method for the tolerance allocation problem. Artif. Intell. Eng., 14: 133-141.

Chiesi, F. and L. Governi, 2003. Tolerance analysis with eM-TolMate. J. Comput. Inf. Sci. Eng., 3: $100-105$.

Chiong, R. and O.K. Beng, 2007. A comparison between genetic algorithms and evolutionary programming based on cutting stock problem. Eng. Lett., 14: 1-6.

Choi, R., M.H. Park and E. Salisbury, 2000. Optimal tolerance allocation with loss functions. J. Manuf. Sci. Eng., 22: 529-535.

Ghiduk, A.S. and M.R. Girgis, 2010. Using genetic algorithms and dominance concepts for generating reduced test data. Informatica, 34: 377-385.

Goldberg, D.E., 1989. Genetic Algorithms in Search Optimization and Machine Learning. Addison Wesley Publishing Co., Reading, Mass achutes.

Hahn G.J. and S.S. Shapiro, 1994. Statistical Models in Engineering. Wiley-Interscience, New York Pages: 376.

Hossin, M., M.N. Sulaiman, A. Mustapha, N. Mustapha and R.W. Rahmat, 2011. OAERP: A better measure than accuracy in discriminating a better solution for stochastic classification training. J. Artif. Intell., 4: 187-196.

Iannuzzi, M. and E. Sandgren, 1994. Optimal tolerancing: The link between design and manufacturing productivity. Proceedings of the ASME Design Theory and Methodology Conference, Volume. 68, September 1994, Minneapolis, MN, USA.

Kanai, S., M. Onozuka and H. Takahashi, 1995. Optimal tolerance synthesis by genetic algorithm under the machining and assembling constraints. Proceedings of the 4th CIRP Seminar on Computer Aided Tolerancing, April 5-6, 1995, Tokyo, Japan, pp: 263-282. 
Kopardekar P. and S. Anand, 1995. Tolerance allocation using neural networks. Inter. J. Adv. Manufacturing Technol., 10: 269-276.

Kumar, R.S., N. Alagumurthi and R. Ramesh, 2009. Optimization of design tolerance and asymmetric quality loss cost using pattern search algorithm. Inter. J. Phys. Sci., 4: 629-637.

Lee, J., 1993. Tolerance optimization using genetic algorithm and approximated simulation. Ph.D. Thesis, The University of Michigan, MI.

Mao, J., Y. Cao, H. Ching and R. Du, 2009. Fuzzy tolerancing based on available manufacturing resources. Comput. Aided Des. Appl., 6: 253-261.

Mukhopadhyay, D.M., M.O. Balitanas, A.A. Farkhod, S.H. Jeon and D. Bhattacharyya, 2009. Genetic algorithm: A tutorial review. Int. J. Grid Distrib. Comput., 2: 25-32.

Muthu, P., V. Dhanalakshmi and K. Sankaranarayanasamy, 2009. Optimal tolerance design of assembly for minimum quality loss and manufacturing cost using metaheuristic algorithms. Int. J. Adv. Manuf. Technol., 44: 1154-1164.

Rajasekera, J.R. and S.C. Fang, 1995. A new approach to tolerance allocation in design cost analysis. Eng. Opt., 4: 283-291.

Ramani, B., S.H. Cheraghi and J.M. Twomey, 1998. CAD-based integrated tolerancing system. Int. J. Prod. Res., 36: 2891-2910.

Rho, S. and S.T. March, 1997. Optimizing distributed join queries: A genetic algorithm approach. Ann. Oper. Res., 71: 199-228.

Singh, P.K., P.K. Jain and S.C. Jain, 2004. A genetic algorithm-based solution to optimal tolerance synthesis of mechanical assemblies with alternative manufacturing processes: Focus on complex tolerance problems. Int. J. Prod. Res., 42: 5185-5215.

Singh, P.K., S.C. Jain and P.K. Jain, 2005. Advanced optimal tolerance design of mechanical assemblies with interrelated dimension chains and process precision limits. Comput. Ind., 56: $179-194$.

Wu C.C., Z. Chen and G.R. Tang, 1998. Component tolerance design for minimum quality loss and manufacturing cost. Comput. Ind., 35: 223-232.

Zhang, H.C. and M.E. Huq, 1992. Tolerancing techniques: The state-of-the-art. Int. J. Prod. Res., 30: 2111-2135.

Zimmermann, D.C., K.C. Yap and T. Hasselmann, 1999. Evolutionary approach for model refinement. Mech. Syst. Signal Proc., 13: 609-625. 